\section{Double-blind trials}

SIR: Oxtoby et al (Journal, November 1989, 155, 700 701) draw attention to the serious difficulties entailed in ensuring that a therapeutic trial achieves the intended ideal of 'double-blindness'. In a doubleblind study the identity of the treatment administered to an individual patient is concealed from both the patient and the assessing clinician, in an attempt to disentangle a 'true' therapeutic effect from any prejudice arising on account of the reputation of a recognised treatment. Ensuring concealment in this way is regarded as particularly important in psychiatric research, in that it would otherwise be impossible to obtain objective evidence (Pocock, 1983) although conversely it may be argued that in some cases in which the aim is purely to provide symptomatic relief, a placebo effect could be construed as validly beneficial.

'Double-blindness' is one of several attributes a trial may exhibit which are unequivocally desirable, other things being equal, but which may not in every instance be feasible. For example, it can be argued that the principle that adequately informed consent on the part of the patient and 'therapeutic equipoise' on the part of the clinician are prerequisites for ethicality would, if taken literally, preclude the majority of studies actually performed. Similarly, not all types of trial can aspire to double-blindness. The comparison of a surgical treatment with a conservative, medical one cannot ethically be made in a double-blind manner. In psychiatry, the 'non-blindness' that can arise from recognition of a drug from its taste or sideeffects is a problem only because recognition leads to imputation of the characteristics the drug is believed to have.

The suggestion that the ability of participants to guess the patients' drug status should be used as a retrospective criterion to exclude certain results is likely to replace one problem with several others.

(a) A phase III drug trial is normally construed as 'pragmatic' in the sense of Schwartz \& Lellouch (1967) and analysed according to the intention-totreat' principle. Retrospective exclusion of some results because in those cases compliance with the principle of 'blindness' could not be obtained is as alien to this scenario as exclusion on account of any other non-compliance or co-intervention.

(b) Some questions could never be answered. In the case of many drugs, such as those with anticholinergic effects, the treatment taken would be 'blind' to the patient in few instances. In the case of less recognisable treatments, retrospective exclusion of a substantial proportion of patients would lead to a serious shortfall in statistical power. (c) Inappropriate significance testing: to advocate discrimination between the discerning patient and the undiscerning by use of a significance test comparing correct guesses or surmises with chance expectation betrays a misunderstanding of the meaning of significance testing and type I and type II errors - the setting of the boundary for such a selection rule is essentially arbitrary, and is made no less so by invoking permutational probabilities under a null hypothesis.

(d) Exclusion of some patients will upset the randomisation and might introduce a selection bias; any observed difference in outcome could be spurious; or conversely, an apparently null difference could be observed because the selection bias masks a true difference. Since we do not know in what respects the undiscerning would differ from the discerning in their susceptibility to (truly) respond to one treatment rather than another, the results of a trial based on the undiscerning could not reasonably be expected to transfer to a target patient population that was not selected in this way.

A more appropriate conclusion to draw from the great difficulty of ensuring 'double-blindness' is that studies sufficiently well planned to be definitive (in particular, heeding the points I raised recently (Newcombe, 1988)), and also as well masked as possible, should be carried out very early in the career of a treatment, before myths concerning its efficacy become widely disseminated. \section{Department of Medical Computing and Statistics University of Wales College of Medicine \\ Heath Park \\ Cardiff CF4 4 XN}

\section{References}

Pocock, S. J. (1983) Clinical Trials. A Practical Approach. (p. 99). New York: Wiley.

SCHWARTZ, D. \& LelLouCh, J. (1967) Explanatory and pragmatic attitudes in therapeutic trials. Journal of Chronic Diseases, 20, 637-648.

NEwCOMBE, R. G. (1988) Evaluation of treatment effectiveness in psychiatric research. British Journal of Psychiatry, 152, 696-697.

\section{Carbamazepine in afiective disorders}

SIR: We expected that the paper by Lusznat et al (Journal, August 1988, 153, 198-204) would give rise to immediate and vigorous comment. As this did not materialise, we wish to record our reservations about the study.

This double-blind trial allocated 54 acutely manic patients to treatment with either carbamazepine or lithium carbonate, and the effects were monitored in 
the acute phase and on a prophylactic basis for up to one year.

Of the 54 patients, 52 received variable doses of neuroleptics during the acute trial, and during the follow-up trial patients were given hypnotics, antidepressants, and neuroleptics when clinically indicated. The carbamazepine group required a higher average dose of neuroleptics in the acute phase, and no comparative information is given about neuroleptic dosage during the follow-up phase. This use of three different types of 'rescue' medication, which was not shown to be equivalent in the two groups, undermines the basis of the comparison made.

Our second reservation centres on the drop-out rate; 40 of the the original 54 patients were no longer in the trial at the end of the 12-month period. This also reduces the weight which can be given to the study. The authors' claim that carbamazepine is more effective as a prophylactic agent than lithium would appear to be poorly founded.

We would appreciate further clarification on the above points, particularly in view of more recent studies which take a less favourable view of carbamazepine as a mood stabiliser (Watkins et al, 1987; Frankenburg et al, 1988).

DENIS J. MURPHY RAJENDER KUMAR

Clinical Headquarters

"Cois Cuain"

140 St Laurence's Road

Clontarf, Dublin 3

\section{References}

Frankendurg, F. R., Tohen, M., Cohen, B. M., el al (1988) Long term response to carbamazepine: a retrospective study. Journal of Clinical Psychopharmacology, 8, 130-132.

Watkins, S. E., Callender, K., Thomas, D. F., et al (1987) The effect of carbamazepine and lithium on remission from affective disorder. British Journal of Psychiatry, 150, 180-182.

SIR: Dr Murphy et al are, in a sense, quite right in what they say. Our sample of patients and method could not have allowed us to answer the question "Is carbamazepine better than lithium in the treatment or prophylaxis of the average manic depressive patient?".

They have, however, missed the point, since that was not the question that we were trying to answer. We simply wanted to see which of the two drugs appeared to be more useful when given in the hurlyburly of ordinary acute psychiatric work and, as a supplementary point, whether one could pick out specific patients particularly likely to respond to either carbamazepine or lithium.
They appear also not to have understood the results that we reported concerning drop-out rates. We had an admittedly uncomfortably high proportion of patients who dropped out through non-compliance, and there were other patients who reached an endpoint for the trial when they relapsed and were readmitted. Dr Murphy et al are combining both groups when they imply that 40 of the original 54 patients were drop-outs.

Most of us have the clinical impression that carbamazepine sometimes works in manic-depressive illness. Our study served to reinforce that impression, and also gave some hints about which types of patients might be expected to do best on carbamazepine (i.e. males with 'textbook' mania). Judging from their address, our critics are writing from a research institute of some sort. One hopes that they will soon get round to doing work with manics, both for its intrinsic value and because it might give them a clearer appreciation of the difficulties involved in doing methodologically pure studies on such a volatile group.

\section{Royal South Hants Hospital}

C. M. H. NunN

R. M. LUSZNAT

Graham Road

Southampton SO9 4PE

\section{Is screening for syphilis justified?}

SIR: We were interested to read the report by Boodhoo on syphilis serology screening in an elderly population (Journal, August 1989, 155, 259-262). In a recent prospective survey of 659 consecutive elderly hospital admissions, we found that $23(3.5 \%)$ had positive serology (Corrado et al, 1989), a similar proportion to that reported by Boodhoo. However, in our study we established the ethnic origin of all patients (to differentiate syphilis from yaws), whether patients showed stigmata compatible with congenital infection, and also whether patients had been previously treated for syphilis in Leeds during the preceding 70 years. Dr Boodhoo has not included this information, which is great import in the interpretation of positive results.

As Lishman (1978) pointed out, syphilis can present with a variety of psychiatric symptoms, and therefore it is difficult to be certain that psychiatric patients with positive serology have not got neurosyphilis. This is particularly true of cognitively impaired patients, and like Dr Boodhoo we had great difficulty in deciding the relevance of positive serology, but only examined the cerebrospinal fluid (CSF) of one patient. It has been suggested that 